\title{
Power-Law Statistics for Avalanches in a Martensitic Transformation
}

\author{
Rajeev Ahluwalia ${ }^{1,2}$ and G. Ananthakrishna ${ }^{2,3}$ \\ ${ }^{1}$ Jawaharlal Nehru Centre for Advanced Scientific Research, Jakkur, Bangalore, India \\ ${ }^{2}$ Materials Research Centre, Indian Institute of Science, Bangalore 560 012, India \\ ${ }^{3}$ Centre for Condensed Matter Theory, Indian Institute of Science, Bangalore 560 012, India
}

\begin{abstract}
We devise a two-dimensional model that mimics the recently observed power-law distributions for the amplitudes and durations of the acoustic emission signals observed during martensitic transformation [Vives et al., Phys. Rev. Lett. 72, 1694 (1994)]. We include a threshold mechanism, long-range interaction between the transformed domains, inertial effects, and dissipation arising due to the motion of the interface. The model exhibits thermal hysteresis and, more importantly, it shows that the energy is released in the form of avalanches with power-law distributions for their amplitudes and durations. Computer simulations also reveal morphological features similar to those observed in real systems.
\end{abstract}

Many spatially extended driven systems naturally evolve to a marginally stable state characterized by avalanches with power-law distributions for their amplitudes and durations reflecting lack of intrinsic length scales and time scales in the system. Such a state is termed as a selforganized critical (SOC) state by Bak et al. [1]. Several physical systems exhibit SOC features: for example, earthquakes [2], acoustic emission from volcanic rocks [3], and stress drops during jerky flow [4], to name a few. Recently, Vives et al. [5], measured the acoustic emission (AE) signals during martensitic transformation of $\mathrm{Cu}-\mathrm{Zn}-\mathrm{Al}$ single crystals under thermal cycling. They reported power-law statistics for the amplitudes and durations of the AE signals during both cooling and heating runs. To the best of our knowledge, there is no strain (or displacement) based model of martensitic transformation which explains these results. Moreover, any prospective model has to take into account the nonequilibrium nature of the hysteresis. Even though extensive theoretical studies exist on martensitic transformations [6-8], the influence of dissipation and defects on hysteresis has received very little attention. Here, we propose a simple phenomenological model which captures the power-law distribution of $\mathrm{AE}$ signals along with the thermal hysteresis of the transformation. Below, we will briefly collect SOC-type features of the martensitic transformation relevant for modeling the system.

Martensitic transformation is a first-order, solid-solid, diffusionless, structural phase transition. On cooling, the unit cell gets distorted [6-8], leading to the nucleation of thin platelike product domains with a twinned structure. This induces internal strains, which in turn induce longrange fields that block the transformation leaving the system in a two phase metastable state. (Note that this implies the existence of a built-in threshold mechanism.) Thus, the amount of the transformed phase is entirely determined by the excess free energy, and an additional undercooling is required for further growth. This implies that thermal fluctuations have little role in the transformation kinetics. Thus, the transformation is athermal and hence the nucleation is athermal, usually occurring at defects such as dislocations $[6,8-10]$. Further, the emission of acoustic energy in the form of bursts implies that inertial effects are important. Indeed, Bales and Gooding, and later Reid and Gooding, [11] studied the importance of including the inertial effects. Vives et al. attribute the mechanism of irreversible release of the elastic energy in the form of avalanches, each of which correspond to the motion of one (or more) interface, to the evolution of the system from one metastable state to another [5]. Since the interface moves at near velocity of sound, there is dissipation associated with it. We include all these features along with the long-range interaction between the transformed domains.

We consider, a 2D square-to-rectangle transition, for which the free energy is usually a function of all three components of strain defined by $e_{1}=\left(\eta_{x x}+\eta_{y y}\right) / \sqrt{2}, e_{2}=$ $\left(\eta_{x x}-\eta_{y y}\right) / \sqrt{2}$, and $e_{3}=\eta_{x y}=\eta_{y x}$, where $\eta_{i j}=$ $\frac{1}{2}\left(\frac{\partial u_{i}}{\partial x_{j}}+\frac{\partial u_{j}}{\partial x_{i}}\right)$ refers to the components of the strain tensor and $u_{i}$ is the displacement field in the direction $i \quad(i=$ $x, y)$. Here, $e_{1}$ is the bulk dilatational strain, $e_{2}$ is the deviatoric strain, and $e_{3}$ is the shear strain. In athermal martensites, deviatoric shear strains play a dominant role in the transformation kinetics [6]. Thus, we consider the deviatoric strain $e_{2}=\epsilon(\vec{r})$ as the principal order parameter since volume changes are small $[6-8,12]$. The effect of other strain components $e_{1}$ and $e_{3}$, i.e., the bulk and shear strain, is accounted phenomenologically by considering a long-range interaction between the deviatoric strains.

We write the rescaled free-energy functional as $F\{\epsilon(\vec{r})\}=F_{L}\{\epsilon(\vec{r})\}+F_{l r}\{\epsilon(\vec{r})\}$, where $F_{L}$ is

$$
F_{L}=\int d \vec{r}\left[f_{l}[\epsilon(\vec{r})]+\frac{D}{2}[\nabla \epsilon(\vec{r})]^{2}-\sigma(\vec{r}) \epsilon(\vec{r})\right],
$$

where $D$ and $\sigma$ are in a scaled form. $F_{l r}$ is an effective long-range term that describes transformation induced strain-strain interactions. In Eq. (1), $f_{l}[\epsilon(\vec{r})]=\frac{\delta T}{2} \epsilon(\vec{r})^{2}-$ $\epsilon(\vec{r})^{4}+\frac{1}{2} \epsilon(\vec{r})^{6}$ is the usual Landau polynomial for a 
first-order transition, where $\delta T=\left(T-T_{c}\right) /\left(T_{0}-T_{c}\right)$ is the scaled temperature. $T_{0}$ is the first-order transition temperature at which the free energy for the product and parent phases are equal, and $T_{c}$ is the temperature below which there are only two degenerate global minima $\epsilon= \pm \epsilon_{M}$. Following Cao et al. [10], the effect of localized defects acting as nucleation sites is simulated by an inhomogeneous stress field, $\sigma(\vec{r})$. This term modifies the free energy $f_{l}$ in a way that renders the austenitic phase locally unstable, leading to the nucleation of the product phase. As mentioned earlier, the physical cause of the long-range interaction is the coherency strain between the parent and the product phases. Such an interaction is also expected to arise due to the coupling of $\epsilon(\vec{r})$ with the other components of the strain order parameter. Recently, an effective long-range interaction has been shown to result, both for the bulk $[13,14]$ and interface [14], between the deviatoric strains if $e_{1}$ and $e_{3}$ are eliminated by imposing (St. Venant) elastic compatibility constraint. In Ref. [14], to describe the effect of the interface between the austenitic-martensite phases, the authors introduce an explicit interface term. Instead, we follow Wang and Khachaturyan [15] who have shown that the interface can be naturally described by accounting for coherency strains at the parent-product interface by a symmetry allowed fourth-order anisotropic long-range interaction in the free energy. Following this, we write the long-range term phenomenologically in the Fourier space as

$$
F_{l r}\{\epsilon\}=\int d \vec{k} B(\vec{k} / k)\left\{\epsilon^{2}(\vec{r})\right\}_{k}\left\{\epsilon^{2}(\vec{r})\right\}_{k^{*}},
$$

where $\left\{\epsilon^{2}(\vec{r})\right\}_{k}$ and $\left\{\epsilon^{2}(\vec{r})\right\}_{k^{*}}$ are the Fourier transform of $\epsilon^{2}(\vec{r})$ and its complex conjugate, respectively. For the square-to-rectangle transformation, the favorable growth directions for the product phase are the habit plane directions [11] and [11]]. In addition, free-energy barriers should be large along the [10] and [01] directions. These features are well captured by the simple kernel $B(\vec{k} / k)=$ $-\frac{1}{2} \beta \theta(k-\Lambda) \hat{k}_{x}^{2} \hat{k}_{y}^{2}$, where $\hat{k}_{x}$ and $\hat{k}_{y}$ are the unit vectors in the $x$ and $y$ directions, $\beta$ is the strength of interaction, and $\Lambda$ in the step function $\theta(k-\Lambda)$ is a cutoff on the interaction range. This kernel incorporates the effect of the interface in a natural way since the cost of growth progressively increases with the transformation in directions where the kernel is positive, which not only aids growth along the habit plane directions but also limits the growth of domains transverse to it. We stress that this is only a simple choice and is not unique. Other kernels with similar orientation dependence will give similar results.

Even though deviatoric strain is the order parameter, basic variables are the displacement fields. Thus, we start with the Lagrangian $L=\mathcal{T}-F$, where $F$ is the total free energy with the kinetic energy $\mathcal{T}$ defined by

$$
\mathcal{T}=\int d \vec{r} \rho\left[\left(\frac{\partial u_{x}(\vec{r}, t)}{\partial t}\right)^{2}+\left(\frac{\partial u_{y}(\vec{r}, t)}{\partial t}\right)^{2}\right]
$$

Here $\rho$ is the mass density. The dissipation associated with the movement of the parent-product interface is represented by the Rayleigh dissipative functional [16]. Further, since deviatoric strains are the dominant ones, the dissipative functional is written entirely in terms of $\epsilon(\vec{r})$, i.e., $R=\frac{1}{2} \gamma \int d \vec{r}\left[\frac{\partial}{\partial t} \epsilon(\vec{r}, t)\right]^{2}$. (This is consistent with the fact that shear and bulk strains are known to equilibrate rapidly and hence do not contribute.) The equations of motion for the displacement fields are

$$
\frac{d}{d t}\left(\frac{\delta L}{\delta \dot{u}_{i}}\right)-\frac{\delta L}{\delta u_{i}}=-\frac{\delta R}{\delta \dot{u}_{i}}, \quad i=x, y .
$$

Using the above equations, we obtain the equation of motion for $\epsilon(\vec{r}, t)$, which after scaling out $\rho$ and $D$ (in terms of rescaled space and time variables) gives

$$
\frac{\partial^{2}}{\partial t^{2}} \epsilon(\vec{r}, t)=\nabla^{2}\left[\frac{\delta F}{\delta \epsilon(\vec{r}, t)}+\gamma \frac{\partial}{\partial t} \epsilon(\vec{r}, t)\right] .
$$

Thus, both $\beta$ and $\gamma$, here, are to be taken as rescaled parameters. The structure of Eq. (5) is similar to that derived in [11] for 1D except for the long-range term.

Simulations are carried out by discretizing Eq. (5) on a $N \times N$ grid using Euler's scheme with periodic boundary conditions. The mesh size of the grid is $\Delta x=1$ and the time step $\Delta t=0.002$. The long-range term is computed using a psuedospectral method: this term is calculated in Fourier space, and then inverse fast Fourier transform is effected to obtain the real space interaction. (We used the cutoff distance $\Lambda=0.2$.) We studied both the single defect and many defect nucleation case. Here, we report results on the multisite case and only mention results on the single site case wherever necessary.

Consider the nucleation and growth for a single quench. We use a random distribution of defects and represent their stress field by $\sigma(\vec{r})=\sum_{j}^{j_{\max }} \sigma_{0}\left(\vec{r}_{j}\right) \exp \left[-\left|\vec{r}-\vec{r}_{j}\right|^{2} / \zeta^{2}\right]$, where $\vec{r}_{j}$ refers to the scaled coordinates of the defect sites and $j_{\max }$ is the total number of defect sites. $\sigma_{0}\left(\vec{r}_{j}\right)$ is taken to be uniformly distributed in the interval $[-0.3,0.3]$. Initially, the system is taken to be in a homogeneous state with $\epsilon(\vec{r}, 0)$ distributed uniformly in the interval $[-0.005,0.005]$. At $t=0$, we switch on the stress field $\sigma(\vec{r})$. Figure 1 shows snapshots of the transformation at $t=10,12,15$, and 50. (Grey regions represent the austenitic phase $\epsilon=0$, and black and white regions represent the two variants.) Nucleation of the product phase occurs with a value $\epsilon \sim \pm 2$ at several locations, where the magnitude of the stress field is sufficient to make the system locally unstable $(t=10)$. We note here that the value of $\epsilon$ for the two variants is larger than that given by $f_{l}[\epsilon(\vec{r})]$ alone $\left(\epsilon_{M} \sim \pm 1.31\right.$ for these parameters $)$ due to the long-range interaction. In a short time, we see the other variant being created adjacent to these nuclei. By $t=12$ (Fig. 1), the structure further develops into twinned arrays,

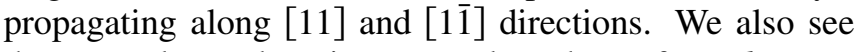
that several new domains are nucleated at a finite distance from the preexisting domains which is consistent with the autocatalytic nucleation mechanism known to operate 

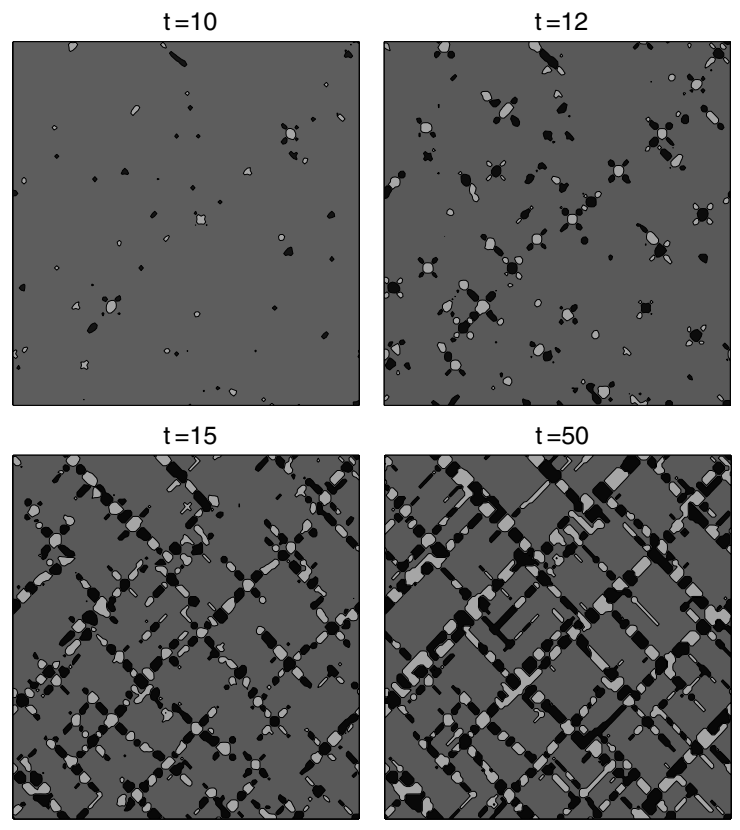

FIG. 1. Snapshots of the morphology of the transformation at $t=10,12,15$, and 50 time steps for multisite nucleation. The parameters values are: $N=128, \delta T=-2.0, \beta=50, \gamma=4$, $\sigma_{0}=[-0.3,0.3]$, and $j_{\max }=164$.

in martensitic transformation [8,9]. Although the new nucleation sites most often coincide with the defect sites, occasionally they are seen in defect-free regions. (Similar observation was made in the single site case also.) This can be attributed to the stress accumulation at these sites as a result of the long-range term due to the preexisting martensitic domains. Note that the twinning is irregular which is again due to the mutual interaction between the various domains. There is very little growth beyond $t=30$. Thin needlelike structures can also be seen to emerge from larger domains $(t=50)$ as reported by Vives et al. [5]. There is also a distribution of domain sizes.

We simulate thermal cycling of the transformation by continuously changing $\delta T$ from +40 to -80 and back, in a duration of $t=1000$ units at a constant rate, for both the heating and the cooling runs. For the reverse cycle, the final configuration of the cooling run is used as the initial configuration. Figure 2 shows the area fraction $\phi_{A}$ versus $\delta T$ for the cooling and heating runs (o). In the cooling run, the transformation starts with a rapid increase in $\phi_{A}$ at about $\delta T_{m s} \sim-2.0$, and is completed by $\delta T_{m f} \sim-59$. In the heating run, the parent phase appears only at $\delta T_{a s} \sim-26.0$ and $\phi_{A}$ decreases almost linearly till the transformation is nearly complete at about $\delta T_{a f} \sim 18$. [For the sake of comparison, we have also shown the single site hysteresis loop by $(\bullet)$.]

The most important feature of the model is that the changes in $\phi_{A}$ are actually jerky which can only be seen on a finer scale. Since the rate of energy dissipated $d E / d t=$ $-2 R(t)$, we calculated $R(t)$ [or $R(\delta T)$ ]. In Fig. 3, we plotted $R(\delta T)$ with the inset showing the enlarged section of the peak which clearly shows that the rate of energy re-

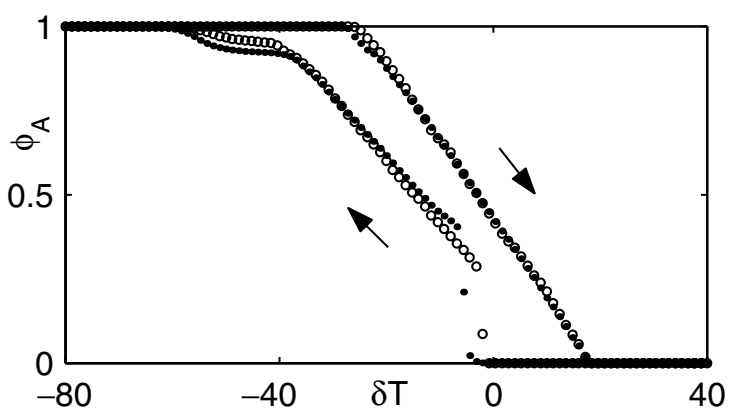

FIG. 2. Area fraction $\phi_{A}$ as a function $t$ for cooling and heating (o) runs for $N=256, \beta=50, \gamma=4, \sigma_{0}=[-0.3,0.3]$, and $j_{\max } \sim 1 \%$ of $N^{2}$. $(\bullet)$ corresponds to the single site case.

lease occurs in bursts consistent with the acoustic emission studies [5]. (Latent heat also shows a pattern similar to Fig. 3 [17].) Further, the distribution of the amplitudes of $R(t)$ denoted by $R_{A}$ has a tendency to approach a power-law $D_{R}\left(R_{A}\right) \sim R_{A}^{-\alpha_{R}}$ with $\alpha_{R} \sim 2.6[(\circ)$ in Fig. 4. We have also shown the single site results by $(\bullet)$.] Similarly, the distribution $D_{R}(\Delta t)$ of the durations $\Delta t$ of the energy bursts scales with $\Delta t$ given by $D_{R}(\Delta t) \sim \Delta t^{-\tau_{R}}$ with $\tau_{R} \sim$ 3.2. Although the scaling regime is almost identical to $D_{R}\left(R_{A}\right)$, we find that the scatter is slightly more in this case. (This is true of experimental results as well [5] and in SOC models [1].) The conditional average [18] of $R_{A}$, for a given $\Delta t$ denoted by $\left\langle R_{A}\right\rangle_{c}$ behaves as $\left\langle R_{A}\right\rangle_{c} \sim \Delta t^{x_{R}}$. We get the exponent value $x_{R} \sim 1.36$. We also find that the scaling relation $\tau_{R}=x_{R}\left(\alpha_{R}-1\right)+1$ is satisfied quite well. In experiments, however, one measures the amplitude of the $\mathrm{AE}$ signals $A_{\mathrm{AE}}$, i.e., $R_{A} \sim A_{\mathrm{AE}}^{2}$. Using the relationship between the two joint probability distributions $D\left(R_{A}, \Delta t\right) \propto D\left(A_{\mathrm{AE}}, \Delta t\right) / A_{\mathrm{AE}}$, it can be easily shown that $\alpha_{R}=\left(\alpha_{\mathrm{AE}}+1\right) / 2$, where $\alpha_{\mathrm{AE}}$ is the exponent corresponding to $A_{\mathrm{AE}}$. The other two exponents remain unchanged. Using the experimental values [5] $\left(\alpha_{\mathrm{AE}} \sim 3.8\right.$, $\tau_{\mathrm{AE}} \sim 3.6$, and $x_{\mathrm{AE}} \sim 1$ ), we see that $\alpha_{R} \sim 2.4$. Thus, we see that our values are in reasonable agreement with experiments, considering the fact that real systems are 3D. It must be stated here that in 3D even the number of variants are generally more, and one also expects that the

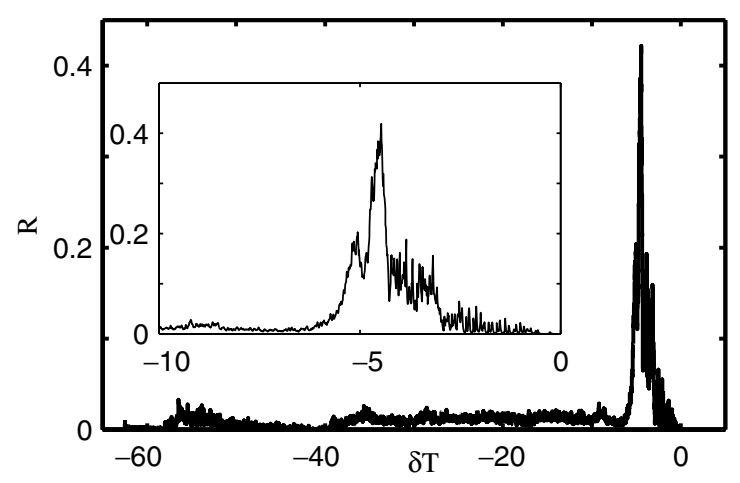

FIG. 3. $R(\delta T)$ as a function of $\delta T$ in the cooling run for $N=256, \beta=50, \gamma=4, \sigma_{0}=[-0.3,0.3]$, and $j_{\max } \sim 1 \%$ of $N^{2}$. The inset shows the fine structure of the peak. 


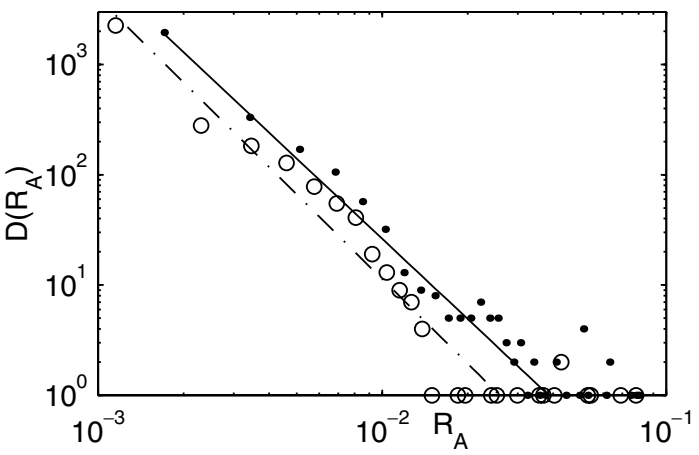

FIG. 4. A plot of $D\left(R_{A}\right)$ versus $R_{A}$ for the same parameter values as in Fig. 3 for the multisite case [(०) connected by a dashed line] with $\alpha=2.6$. ( $\bullet)$ with a continuous line shows the single site case with $\alpha=2.5$.

mechanisms operating in 3D cannot be fully accounted for in $2 \mathrm{D}[8,15]$. Similar analysis of $R(t)$ for the heating run shows more scatter in the distributions than that for the cooling run.

In conclusion, the fact that the energy release occurs in the form of bursts, with power-law statistics for the avalanches, is well captured by the model. In addition, the model shows hysteresis under thermal cycling. The power-law statistics can be attributed to the fact that we have included important ingredients of SOC dynamics, namely, the threshold dynamics, dissipation, the generation of a large number of metastable states during the transformation, and a relaxation mechanism for the stored energy. This relaxation time scale corresponds to the inertial time scale, as can be inferred from the fact that the basic variables are the displacement fields, which in turn set the limit on the fastest time scale. Indeed, from our simulations we find that the interface movement occurs at time scales of a few units of (scaled) time. Compared to this the driving force increases with temperature at a slow pace. The important feature of inducing a large number of metastable states during cooling or heating runs is the long-range interaction between the transformed domains, as can be seen from the following reasoning. We note that the value of this term at any location is the result of the superposition of the contributions arising from the spatial distribution of the already transformed domains, which in turn leads to a complex terrain of local barriers (metastable states). These self-generated (transformation induced) local thresholds, at a given time, must be overcome by an increase in the driving force arising from the slow cooling (or heating). We note that, once a local barrier is overcome, part of the driving force goes toward creating a new twin and the rest is dissipated in the form of a burst of energy (due to the advancing interface). The fact that long-range interaction is at the root of creating the local thresholds is further supported by the fact that we find a power-law distribution even in the single site nucleation case [see (•) in Fig. 4]. The presence of defect sites only triggers the initial nucle- ation process. This must be contrasted with disorder based Ising models [19] which also produce power-law statistics for avalaches and field induced hysteresis. However, by subjecting the samples to repeated thermal cycling, Vives et al. have verified that, in martensite transformation, it is the dynamical (transformation induced) disorder that is at the root of the avalanches. In this sense, our model is the first to capture both the thermal hysteresis and the jerky nature of the transformation based on dynamical disorder and is independent of quenched disorder.

We thank Ms. M.S. Bharathi for the analysis of the data. Financial support from JNCASR for carrying out this project is gratefully acknowledged.

[1] P. Bak, C. Tang, and K. Wiesenfeld, Phys. Rev. A 38, 364 (1988); Phys. Rev. Lett. 59, 381 (1987).

[2] C. F. Richter, Ann. Geophys. 9, 1 (1956).

[3] P. Diodati, F. Marchesoni, and S. Piazza, Phys. Rev. Lett. 67, 2239 (1991).

[4] G. Ananthakrishna, S. J. Noronha, C. Fressengeas, and L. P. Kubin, Phys. Rev. E 60, 5455 (1999).

[5] E. Vives, J. Ortin, L. Manosa, I. Rafols, R. Perez-Magrane, and A. Planes, Phys. Rev. Lett. 72, 1694 (1994); E. Vives, I. Rafols, L. Manosa, J. Ortin, and A. Planes, Phys. Rev. B 52, 12644 (1995); See also Ll. Carrillo et al., Phys. Rev. Lett. 81, 1889 (1998).

[6] See J. W. Christian, G. B. Olson, and M. Cohen, J. Phys. C 8, 3 (1995); P. C. Clapp, J. Phys. C 8, 11 (1995); G. B. Olson, Mater. Sci. Eng. A 273-275, 11 (1999). Acoustic emission is considered an important feature of proper martensites.

[7] A. L. Roitburd and G. V. Kurdjumov, Mater. Sci. Eng. 39, 141 (1979); A. L. Roitburd, Mater. Sci. Eng. A 273-275, 1 (1999).

[8] A. G. Khachaturyan, Theory of Structural Transformations in Solids (Wiley, New York, 1983).

[9] P. L. Ferraglio and K. Mukherjee, Acta Metall. 22, 835 (1974).

[10] W. Cao, J. A. Krumhansl, and R. J. Gooding, Phys. Rev. B 41, 11319 (1990).

[11] G. S. Bales and R. J. Gooding, Phys. Rev. Lett. 67, 3412 (1991); A. C. E. Reid and R. J. Gooding, Phys. Rev. B 50, 3588 (1994).

[12] A. E. Jacobs, Phys. Rev. B 31, 5984 (1985).

[13] S. Kartha, J. A. Krumhansl, J.P. Sethna, and L. K. Wickham, Phys. Rev. B 52, 803 (1995).

[14] S. R. Shenoy, T. Lookman, A. Saxena, and A. R. Bishop, Phys. Rev. B 60, R12537 (1999).

[15] Y. Wang and A. G. Khachaturyan, Acta Mater. 45, 759 (1997).

[16] L. D. Landau and E. M. Lifschitz, Theory of Elasticity (Pergamon, Oxford, 1986), 3rd ed.

[17] C. Auguet, E. Cesari, and Ll. Manosa, J. Phys. D 22, 1712 (1989).

[18] I. Rafols and E. Vives, Phys. Rev. B 52, 12651 (1995).

[19] J. P. Sethna et al., Phys. Rev. Lett. 70, 3347 (1993); E. Vives and A. Planes, Phys. Rev. B 50, 3839 (1994); A. Magni, Phys. Rev. B 59, 985 (1999). 